\title{
A prospective study on combined lymphedema surgery: Gastroepiploic vascularized lymph nodes transfer and lymphaticovenous anastomosis followed by suction lipectomy
}

\author{
Giuseppe Di Taranto MD ${ }^{1,2}$ (1) | Alberto Bolletta MD ${ }^{1,3}$ ( ) | Shih-Heng Chen MD \\ Luigi Losco MD ${ }^{1,5}$ (i) | Rossella Elia MD ${ }^{1,6}$ (i) | Emanuele Cigna MD \\ Corrado Rubino $\mathrm{MD}^{3}$ | Diego Ribuffo $\mathrm{MD}^{2}$ | Hung-Chi Chen MD, PhD, FACS ${ }^{1}$
}

\footnotetext{
${ }^{1}$ Department of Plastic Surgery, China Medica University Hospital, Taichung, Taiwan

${ }^{2}$ Department of Plastic Surgery, University of Rome La Sapienza Department of Surgery, Rome, Italy

${ }^{3}$ Department of Medical, Surgical and Experimental Sciences, Plastic Surgery Unit, Universita degli Studi di Sassari, Sassari, Italy

${ }^{4}$ Department of Plastic Surgery Taoyuan, Chang Gung Memorial Hospital Linkou Main Branch, Taoyuan, Taiwan

${ }^{5}$ Dipartimento di Ricerca Traslazionale e delle Nuove Tecnologie in Medicina e Chirurgia, Università degli Studi di Pisa Facolta di Medicina e Chirurgia, Pisa, Italy

${ }^{6}$ Universita degli Studi di Bari Dipartimento Emergenza e Trapianti di Organi, Division of Plastic and Reconstructive Surgery, Bari, Italy

Correspondence

Hung-Chi Chen, Department of Plastic Surgery, China Medical University Hospital, 2 Yuh Der Road, Taichung 404, Taiwan. Email: d19722@mail.cmuh.org.tw
}

\begin{abstract}
Background: There is no consensus on the appropriate treatment of lymphedema. Proposed techniques include lymphaticovenous anastomosis (LVA), vascularized lymph nodes transfer (VLNT), and suction lipectomy (SL). The benefit of combined procedures has also been postulated. In this prospective study, a combined protocol is proposed as an alternative to single-procedure strategies.

Methods: Between January 2016 and October 2018, we enrolled patients with secondary lymphedema of lower limbs, stage II-III according to the International Society of Lymphology, progressive swelling and skin tonicity $>60$. Thirty-seven consecutive patients were dichotomized into group I, undergoing VLNT, and group II undergoing VLNT and LVA. Gastroepiploic lymphnode flap was harvested through laparoscopy, and in the same operation, LVAs were performed in group II on the basis of indocyanine green lymphography and patent blue findings. Two weeks later, SL was performed in all the patients. Patients were prospectively evaluated through clinical examination, circumference measurement, and skin tonicity.

Results: The average follow-up was $2 \pm 0.8$ years. The first consecutive 21 patients were treated with VLNT followed by SL. The next 16 patients underwent combined VLNT and LVA, followed by SL. A mean of 2.4 LVAs were performed. A significant difference in the postoperative circumference measurements was found overall $(p<.05): 52.6 \pm 18.9$ above the knee, $42.9 \pm 25$ below the knee, $36.2 \pm 37$ at foot. The postoperative tonicity dropped by $12.7 \pm 6.3 \%(p<.05)$. The episodes of cellulitis significantly decreased to $0.1 \pm 0.3(p<.05)$.

Conclusions: LVA, VLNT, and SL can be integrated together in a combined approach, in synergy to enhance the outcomes.
\end{abstract}

\section{1 | INTRODUCTION}

Lymphedema affects over 250 million people all over the world (Rockson \& Rivera, 2008). Primary lymphedema is a consequence of genetic or developmental abnormalities, whereas secondary 
lymphedema results from a variety of different causes including malignancy, radiation or surgical treatment, infection or trauma affecting the lymphatic system (Allen \& Cheng, 2016; Lo Torto et al., 2018; Rockson \& Rivera, 2008). Whatever the cause, this results in a specific series of events that starts with the accumulation in the interstitial space of fluid rich in proteins, causing inflammation and eventually fibrosis and adipose deposition in the subcutaneous tissues (Avraham et al., 2010; Brorson, 2004; Cuzzone et al., 2014; Lo Torto et al., 2018; Zampell et al., 2012). This process further distresses the lymphatic system in a degenerating cycle, with worsening of symptoms and patient's condition, eventually impeding daily activities (Allen \& Cheng, 2016).

In the early stages of pathological process, conservative and nonsurgical treatment can be advised, but in more advanced stages the surgical treatment becomes necessary (Bolletta, Di Taranto, Chen, et al., 2020; Ciudad et al., 2019; Di Taranto, Chen, Elia, et al., 2020). Early proposed surgical procedures were often invasive and disfiguring. In contrast, recently described surgical techniques have proved to be less invasive and yet still effective in reducing limb volume together with the risk of complications, like cellulitis, or need for successive treatment (Fernández Peñuela, Casaní Arazo, \& Masiá Ayala, 2019).

Today these procedures, which include lymphatic-venous anastomosis (LVA), vascularized lymph node transfer (VLNT), and lymphaticlymphatic bypass are becoming increasingly popular (Di Taranto, Elia, Amorosi, et al., 2018; Yamamoto \& Koshima, 2015; Yamamoto, Yoshimatsu, \& Yamamoto, 2016). Based on the hypothesis that transferred lymph nodes will behave as a new pump helping the deteriorated lymphatic system (Lin et al., 2009), the VLNT consists of the transplantation of lymph node-containing tissue together with artery and vein from a donor site to the affected limb (Becker, Assouad, Riquet, \& Hidden, 2006; Cheng, Huang, Huang, et al., 2012; Saaristo et al., 2012; Viitanen, Mäki, Seppänen, Suominen, \& Saaristo, 2012). The lymphatic fluid is then conveyed through the transferred lymph nodes and the newly anastomosed venous route (Cheng, Loh, \& Lin, 2018; Ito, Zelken, Yang, Lin, \& Cheng, 2016).

LVA consists of performing multiple microsurgical anastomosis between collecting lymphatics and small sized veins in the affected limb. This procedure can be repeated at different levels of the limb (Hayashi, Hayashi, Yoshimatsu, \& Yamamoto, 2018; Seki et al., 2019). Both VLNT and LVA have proved to be effective treatment measures in secondary lymphedema (Allen \& Cheng, 2016; Loh, Wu, Nguyen, et al., 2017; Qiu, Chen, \& Cheng, 2014).

Nevertheless, there is no consensus on an appropriate standardized protocol for the treatment of lymphedema (Loh et al., 2017). Over the past decade, a flood of studies on lymphedema surgery techniques has been released, reporting conflicting outcomes (Cheng, Chang, Masia, \& Koshima, 2020; Ciudad et al., 2017; Maeda et al., 2018; Yamamoto et al., 2016; Yamamoto, Yoshimatsu, \& Yamamoto, 2016). Owing to the lack of evidence, each author apply his personal approach, presenting interesting data and promising results, with a grade of reluctance to other techniques. Recently, a combined tailored approach, merging different procedures has been proposed as an alternative to single-surgery strategies (Chang, Masia, Garza, Skoracki, \& Neligan, 2016; Cheng et al., 2020; Loh et al., 2017; Masià, Pons, \& Rodríguez-Bauzà, 2016).

The purpose of this study is to evaluate our comprehensive protocols for lymphedema and compare the outcomes between the treatment with VLNT alone followed by extensive suction lipectomy (SL) and the combined treatment with VLNT and LVA followed by extensive SL.

\section{I PATIENTS AND METHODS}

Between January 2016 and October 2018, patients presenting with lymphedema were enrolled at our Institution. Among them, we selected patients diagnosed for secondary lymphedema of the lower limbs. Clinical evaluation, and clinical staging of lymphedema were performed in all cases according to the International Society of Lymphology (ISL) (International Society of Lymphology, 2013).

Inclusion criteria were secondary lymphedema of lower limbs, stage II-III, progressive swelling of the limb regardless of decongestive therapy and skin tonicity above 60 (Bolletta et al., 2020; Chen, O'Brien, Pribaz, \& Roberts, 1988; Di Taranto et al., 2020; Di Taranto, Chen, Elia, et al., 2019). Exclusion criteria were primary lymphedema, lymphedema of the upper limbs, previous lymphedema-related or other surgeries of the involved limb and the presence of spontaneous ulcer with lymph leakage. Eligible patients were offered reconstruction through two combined protocols: VLNT followed by SL, or VLNT and LVA followed by SL.

Basing on the inclusion criteria, eligible patients were enrolled upon signing a written informed consent document. Our study was performed with respect to the ethical standards of the Declaration of Helsinki and the protocol was approved by the Ethics Committee of China Medical University (CMUH106-REC1-111[CR1]).

This study was designed as a pilot study to validate our protocol combining LVA and VLNT followed by SL and we applied a simple criteria for dichotomize our population, as previously described (Casella, Di Taranto, Marcasciano, et al., 2019; Fino, Di Taranto, Toscani, \& Scuderi, 2016; Tarallo et al., 2018). Patients were selected from among those diagnosed for lymphedema at our Institution between January 2016 and October 2018. The first consecutive patients enrolled in the study in the first time frame, between 2016 and 2017, were treated with VLNT followed by SL (group I), as described in other reports from our group (Bolletta et al., 2020; Ciudad et al., 2017; Di Taranto et al., 2020). This group was the control group of our study or VLNT group. The following consecutive patients enrolled in 2018 were treated with a different approach involving combined LVA and VLNT followed by SL (group II).

Patients were evaluated 1 month before surgery and in the postoperative period through clinical examination, circumference measurement, skin tonicity, and photographic documentation. Data of all the surgeries were prospectively collected in a digital database, accounting for demographics and surgical characteristics, medical history, complications and outcomes. The circumference reduction rate 
(CRR) was used to compare preoperative and postoperative findings. The CRR was defined as the difference between the preoperative circumferences of the affected and healthy limbs minus the difference between the postoperative circumferences of the affected and healthy limbs, divided by the preoperative difference, $\times 100$, as previously described (Di Taranto et al., 2020; Maruccia et al., 2019).

Patients were followed-up once a week for the first month after surgery and then follow-up points were scheduled every month for the first year, and then every 6 months. Tonicity assessment was performed at 1-year follow-up and evaluated with a tonometer with a central plunger at two levels, as previously described (Bolletta et al., 2020; Di Taranto et al., 2020).

\section{1 | Surgical technique}

In both groups, VLNT was performed; only the group II underwent LVA in the same surgery, just before the VLNT. For LVA incision sites were determined on the basis of preoperative indocyanine green lymphographic (ICG) (Figure 1). After injection at the first interdigital space, the skin was massaged and the pattern of the lymphatic network was marked. Intraoperatively, patent blue was injected at the same sites, in order to better identify the lymphatic vessels. Stained subdermal lymphatic vessels appeared within $1 \mathrm{~min}$ and continued proximally, assisting the surgery (Ayestaray, Bekara, \& Andreoletti, 2013; Yap et al., 2009). Incisions were performed under magnification and preferentially placed over linear lymphatic pattern, which suggested healthy, functioning lymphatic vessels, just distal to an area of dermal backflow (Pons et al., 2019; Yamamoto, Yamamoto, Fuse, Narushima, \& Koshima, 2018). According to previous reports, we located our incisions and performed LVAs on the most proximal aspect of a linear pattern before an area of dermal backflow (Kaciulyte et al., 2019; Yamamoto et al., 2018). The incision was placed distal to the area of dermal backflow where it was possible to locate a lymphatic vessel characterized by linear pattern. In this setting, we preferred to perform end-to-end anastomosis owing to disruption of the proximal lymphatic network (Yamamoto et al., 2018) (Figure 2). In the absence of a defined pattern, incisions were placed

FIGURE 1 Preoperative view of a patient affected by left lower limb lymphedema studied with indocyanine green lymphography. Circumference measurement was performed at different levels (black lines and numbers) and showed great differences between the limbs. On the left, the frontal view showing the area of dermal backflow, marked in blue. On the right, a lateral view displaying areas of linear pattern, marked with a blue dotted line
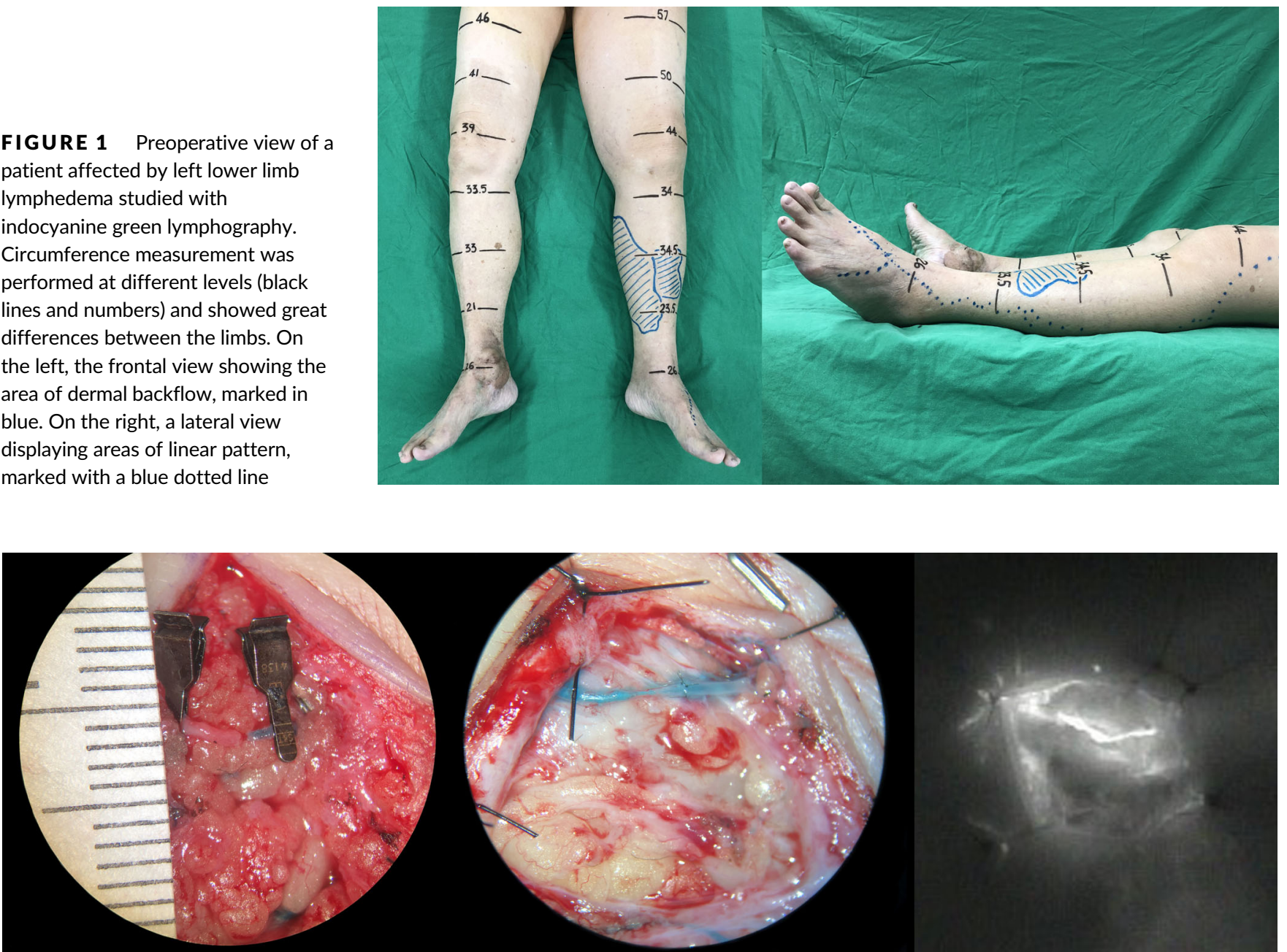

FIGURE 2 On the left, suitable lymphatic vessel and vein are identified and prepared. On the right, lymphatico-venous anastomosis is performed in end-to-end fashion with 11-0 nylon and supermicrosurgical instruments. The flow through LVA is checked both with patent blue dye (middle) and ICG (right) 
along the anatomic courses of the superficial veins, preferably along the greater saphenous vein. Whenever healthy lymphatic vessels and size-matched veins were present, LVAs were performed in an end-toend anastomosis technique using 11-0 nylon and supermicrosurgical instruments. LVAs were performed only on the dorsal aspect of the foot up to the level of lower leg, which was spared in the following lipectomy procedure, in order to avoid any injury to the anastomosis.

The lymphnode flap, based on right gastroepiploic artery, was harvested from the omentum through laparoscopy, as previously described (Ciudad, Manrique, et al., 2017; Di Taranto et al., 2020). Briefly, the abdomen was accessed with three ports and a small omental window was created between the stomach and the transverse colon. Meticulous dissection was conducted and the left gastroepiploic vessels were then identified and carefully ligated. Using sharp and blunt dissection, the right gastroepiploic artery and vein were exposed and individually ligated.

After the harvesting, under microscope magnification, the course of the gastroepiploic vessels was identified and careful dissection was performed at the junction center of the omental flap in order to divide the flap into two similar lymph node flaps. The flaps were then inset at two different levels for each extremity. The flap was anastomosed in the lower extremity at the medial aspect of the ankle using the medial plantar vessels and at the popliteal fossa using the medial sural artery and venae comitantes as recipient vessels (Figure 3 ). The lymph

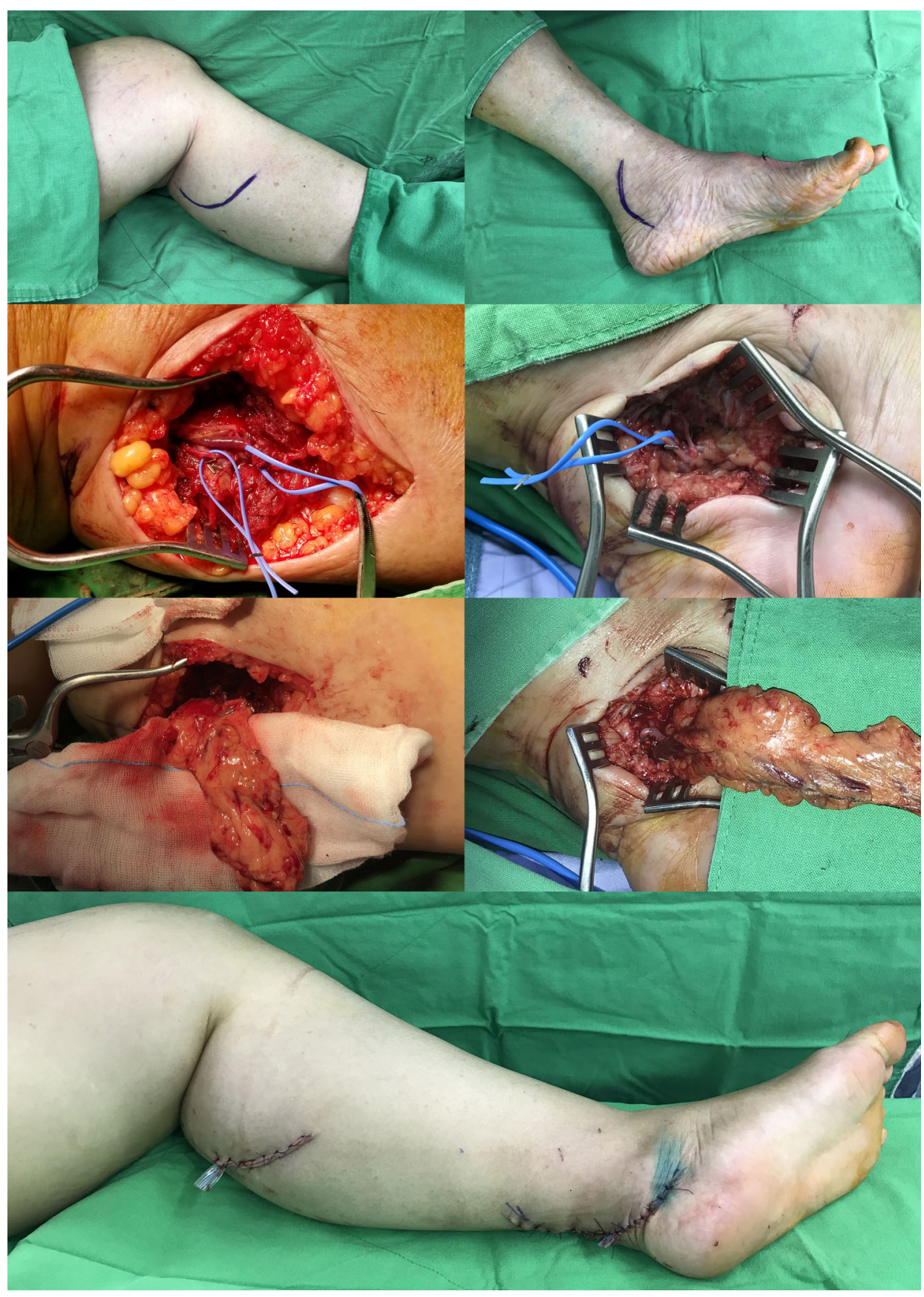

FIGURE 3 Operative steps for gastroepiploic vascularized lymph node transfer. Upper panels: preoperative markings at medial ankle and popliteal fossa. Left central panels: medial sural vessels were exposed and dissected and the flap anastomosed in and end-to-end fashion at the popliteal fossa. Right central panels: medial plantar vessels are exposed and dissected and the flap anastomosed in and end-to-end fashion. Panel below: postoperative view 
node flaps were covered using local flaps or split thickness skin graft harvested from the upper thigh in case of a bulky flap. Flap monitoring was done clinically and with hand-held Doppler (Kaciulyte et al., 2019).

Two weeks after the VLNT, extensive lipectomy was performed, sparing the sites of VLNT and LVA, in order to avoid any injury to the anastomoses and the transferred lymphnodes (Brorson, 2004; Brorson, 2016).

Vacuum drains were applied and removed the day after surgery. Compression garments were applied immediately after surgery.

\section{2 | Statistical analysis}

SPSS software (IBM Corp., Armonk, NY) was used for statistical analysis. The Shapiro-Wilk test was used to verify normal distribution of continuous variables. Differences between groups were analyzed by chi-square test, Fisher's exact probability test, sign test, paired or unpaired $t$ test, or Mann-Whitney test, as appropriate. Statistical significance was defined as $p<.05$.

\section{3 | RESULTS}

Hundred and forty-one patients presented for lymphedema at our Institution. Among them, 37 patients, meeting the inclusion criteria, were enrolled in the study. The first consecutive 21 patients underwent VLNT (group I) followed by extensive SL and the next consecutive patients underwent the combined treatment, VLNT and LVA (group II) followed by extensive SL.

Table 1 describes the characteristics of the patients and surgeries. The mean age of patients was $51.7 \pm 16.3$ years. The two groups were similar in term of BMI, age, comorbidities, cause of lymphedema, lymphedema grade, and sex distribution. However, the groups differed for the length of the follow-up $(p<.05)$.

Mean BMI was assessed as $26.7 \pm 3.2 \mathrm{~kg} / \mathrm{m}^{2}$. Eight patients presented Stage III lymphedema and 29 patients Stage II lymphedema. Patients reported an average number of 1.4 preoperative episodes of cellulitis per year.

The patients successfully underwent the VLNT, involving laparoscopic free omental lymphatic flap for covering the defect. Only the 16 patients enrolled between January and October 2018 underwent LVA before VLNT in the same operation. In this group a mean of 2.4 LVA was performed. Two weeks after the lymphatic surgery, all the patients underwent suction-assisted lipectomy. The mean volume of liposuction in patients of the VLNT and LVA group was 2,484 $\pm 654 \mathrm{cc}$, while the mean volume of liposuction in the VLNT group was $2,528 \pm 604 \mathrm{cc}$. No statistically significant difference was found regarding this aspect $(p=.841)$.

All the patients healed uneventfully. Only in three cases, we reported partial loss of the skin graft adjacent to the lymph node flap at the ankle. These patients underwent debridement and healed

TABLE 1 Characteristic of patients, surgeries, and outcomes

\begin{tabular}{|c|c|c|c|}
\hline \multicolumn{4}{|l|}{ Demographics } \\
\hline Group & $\operatorname{VLNT}(n=21)$ & VLNT and LVA $(n=16)$ & All population $(n=37)$ \\
\hline Age (years: Mean $\pm S D$ ) & $51.9 \pm 16.8$ & $51.4 \pm 16.1$ & $51.7 \pm 16.3$ \\
\hline \multicolumn{4}{|l|}{ Sex } \\
\hline Male (n) & 6 & 2 & 8 \\
\hline Female $(n)$ & 15 & 14 & 29 \\
\hline $\mathrm{BMI}\left(\mathrm{kg} / \mathrm{m}^{2}:\right.$ Mean $\left.\pm S D\right)$ & $27.1 \pm 3.2$ & $26.2 \pm 3.2$ & $26.7 \pm 3.2$ \\
\hline Hypertension & 4 & 4 & 8 \\
\hline Diabetes & 4 & 2 & 6 \\
\hline Preoperative episodes of cellulitis (mean $\pm S D$ ) & $1.4 \pm 2$ & $1.4 \pm 1.3$ & $1.4 \pm 1.7$ \\
\hline \multicolumn{4}{|l|}{ Lymphedema characteristics } \\
\hline \multicolumn{4}{|l|}{ Stage of lymphedema } \\
\hline Il stage $(n)$ & 16 & 13 & 29 \\
\hline III stage $(n)$ & 5 & 3 & 8 \\
\hline \multicolumn{4}{|l|}{ Etiology } \\
\hline Gynecological cancer & 15 & 11 & 26 \\
\hline Urologic cancers & 3 & 2 & 5 \\
\hline Radiotherapy & 2 & 1 & 3 \\
\hline Melanoma & 1 & 2 & 3 \\
\hline \multicolumn{4}{|l|}{ Characteristics of the surgeries } \\
\hline Number of LVA (mean $\pm S D$ ) & 0 & $2.4 \pm 0.5$ & $1 \pm 1.2$ \\
\hline Follow-up (years) & $2.5 \pm 0.8^{*}$ & $1.4 \pm 0.3^{*}$ & $2 \pm 0.8$ \\
\hline
\end{tabular}

${ }^{*} p<.05$. 
uneventfully by secondary healing. No other postoperative complication occurred. The patients did not report any postoperative donor site-related complications or dyspepsia and disfunction of intestinal motility.

The average follow-up was $2 \pm 0.8$ years. In the overall population, the mean circumference reduction rate was $52.6 \pm 18.9,42.9$ $\pm 25,19.2 \pm 34.4$, and $36.2 \pm 37 \%$ above the knee, below the knee, above the ankle, and at the foot level, respectively, despite of bulk of the flap (see Table 2). Statistical analysis found a significant difference in the circumference measurements above the knee, below the knee and at the foot level from the preoperative to the postoperative period in the affected limb $(p<.05)$. At 1-year follow-up, the VLNT and LVA group scored a grater circumference reduction rate at all levels in comparison to the VLNT group (Figure 4). Nevertheless, no statistically significant difference was found. The tonicity dropped by
$12.7 \pm 6.3 \%$ from the preoperative period in the overall population $(p<.05)$. Over the follow-up, a significant decrease in the episodes of cellulitis from the preoperative period was noted $(p<.05)$.

\section{4 | DISCUSSION}

In this study, we evaluated our comprehensive protocols for lymphedema by comparing the outcomes between the treatment with VLNT and extensive SL alone and a new combined treatment with VLNT, LVA and extensive SL.

Up to date, a standardized approach for the treatment of lymphedema has not been agreed. Many techniques have been proposed and their effectiveness studied in patients diagnosed with lymphedema at different stages. As other authors have suggested, the LVA

TAB LE 2 Outcomes at 1-year follow-up after the combined protocol

\begin{tabular}{|c|c|c|c|c|c|}
\hline \multicolumn{6}{|l|}{ Outcomes } \\
\hline Group & VLNT $(n=21)$ & VLNT and LVA $(n=16)$ & $p$ & All population $(n=37)$ & $p^{*}$ \\
\hline \multicolumn{6}{|l|}{ Mean circumference reduction rate $(\%$ : Mean $\pm S D)$} \\
\hline Above the knee & $47.9 \pm 15.4$ & $58.8 \pm 21.7$ & .08 & $52.6 \pm 18.9$ & $<.01$ \\
\hline Below the knee & $37.1 \pm 22.2$ & $50.5 \pm 27.1$ & .10 & $42.9 \pm 25$ & $<.01$ \\
\hline Above the ankle & $18.6 \pm 35.6$ & $22 \pm 33.9$ & .91 & $19.2 \pm 34.4$ & .42 \\
\hline Foot & $32.8 \pm 26$ & $40.7 \pm 48.4$ & .53 & $36.2 \pm 37$ & .02 \\
\hline Overall reduction of tonicity (\%) & $11.5 \pm 7.3$ & $13.6 \pm 5.5$ & .32 & $12.7 \pm 6.3$ & $<.01$ \\
\hline Postoperative episodes of cellulitis (mean $\pm S D$ ) & $0.1 \pm 0.4$ & $0 \pm 0$ & .39 & $0.1 \pm 0.3$ & $<.01$ \\
\hline
\end{tabular}

Note: $p$ refers to outcomes of VLNT versus VLNT and LVA; $p^{*}$ refers to preoperative versus postoperative overall measurements.

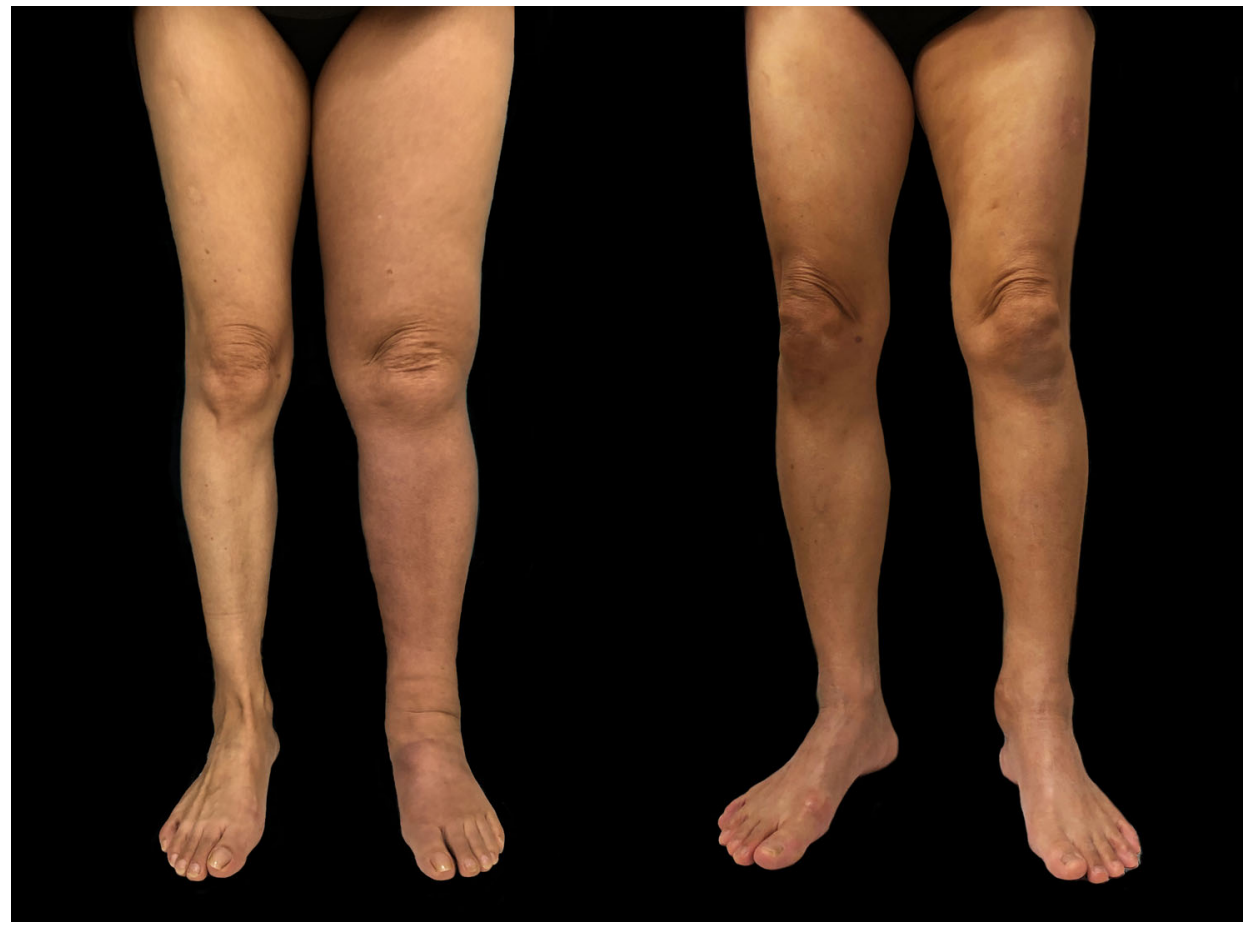

FIGURE 4 Pre-operative (left) and post-operative (right) pictures of a 56-year-old patient affected by left lower limb secondary lymphedema treated with the combined approach of VLNT and LVA followed by suction-assisted lipectomy. The postoperative evaluation was performed 1 year after surgery 
approach proved to be successful in early stages of lymphedema, where lymphatic vessels are still functional (Hayashi et al., 2018; Phillips, Gore, Ramsden, \& Furniss, 2019).

In 2012, Mihara et al. published a study on histological changes occurring to collective lymphatic vessels after lymphadenectomy (Mihara et al., 2012). In their work, they found a strong association between the staging classification of lymphedema by ISL and the composition of lymphatic tissues in terms of changes occurring on the histological level of the collective vessels analyzed. They proved that in advanced stages lymphatic vessels become sclerotic, less functional, and this may be the reason for the reduction in the efficacy of the LVA approach (Ciudad, Agko, et al., 2017; Ciudad, Agko, et al., 2019; Di Taranto et al., 2018; Di Taranto et al., 2020; Inserra et al., 2016). According to Cheng, different stages of lymphedema require different treatments. Thus, LVAs are indicated in patients in early stages (Grade I-II), while VLNT is more advanced cases (Grade II-III and sometimes also IV) (Patel, Lin, \& Cheng, 2015).

In our study, patients additionally treated with LVAs showed improved results in terms of circumference reduction. Indeed, while some patients treated with the combined approach had a circumference reduction rate above average, others did not experience such benefit. It has to be stated though, that in our experience some patients presented with healthier lymphatics of greater caliber, well visualized by ICG; whereas others showed a higher number of lymphatics with a smaller lumen, which appeared impaired by fibrosis. This means that within the same clinical stage, the damage of lymphatics is a continuum; therefore, effectiveness of the additional LVA procedure might depend on the condition of lymphatic vessels and their capacity of benefitting from the anastomosis to the venous system. We strongly believe that a larger study evaluating the outcomes between the single subgroups of lymphedema stage is needed to confirm our results and obtain a statistically significant difference with the combined approach.

VLNT from the gastroepiploic region has been previously described and is considered the first line of treatment for patients with moderate lymphedema in our center (Ciudad, Manrique, et al., 2017). The efficacy of VLNT in reduction of limb circumference has been examined by several authors (Becker et al., 2006; Cheng et al., 2020; Ciudad, Agko, et al., 2017; Ciudad, Manrique, et al., 2017; Maruccia et al., 2019). Our approach was recently evaluated in terms of long-term outcomes and proved to determine a substantial reduction in circumference measurements and episodes of cellulitis (Ciudad, Manrique, Bustos, et al., 2020). In our experience, the combination of the VLNT with extensive therapeutic lipectomy helps reducing the lymphatic load on the newly transferred lymphatic tissue (Agko, Ciudad, \& Chen, 2018; Bolletta et al., 2020; Inserra et al., 2016; Losco et al., 2020). The technique was investigated and found not causing further damage to the lymphatic system, but we avoided treating the areas where the LVAs were performed in order not to accidentally damage them.

Skin tonicity was one of the outcomes evaluated in this study. Subjectively, the softening of the treated limb was perceived by both physicians and patients as greater in patients treated with the combined approach. Although both groups scored a significant reduction of the tonicity from the preoperative period, a slightly higher drop was assessed in the group II. Furthermore, the episodes of cellulitis significantly dropped from the preoperative to the postoperative period, resulting in less days of hospitalization in both groups.

To our knowledge, this is the first study evaluating the effectiveness of the association of VLNT and LVAs in the same surgical procedure in lower limb lymphedema and one of the few with a prospective design comparing two different cohorts of patients. As already stated, several authors prefer to choose the surgical technique according to the staging of the patients. They usually perform LVAs in milder cases and VLNTs in more severe cases (Chang et al., 2016). However, some authors use LVAs as the first treatment line also in more severe cases, then wait to evaluate the results and proceed with VLNT in case of not satisfactory results (Maeda et al., 2018). This is possible since the rationale behind LVAs and VLNT is different, as they have different surgical targets and work with different strategies for approaching the disruption of lymphatic system. LVAs bridge areas where lymphatics are damaged by diverting lymph into the venous system prior to the areas of obstruction. VLNT is supposed to act as a "pump" or a "sponge" absorbing lymph into its nodes and, through the lymphovenous connections in the transplanted tissues, directing it into the venous circulation and promoting lymphoangiogenesis (Cheng et al., 2014). We assumed that LVAs and VLNT can be integrated together in a combined approach to work in synergy, enhancing the outcomes. As previously stated, LVAs and VLNT procedures have already been performed on the same patients as sequential steps in case the first surgical attempt (LVAs) was not successful. Hence, we suggest the association of both procedures in the same surgical stage in order to combine different strategies to treat the disruption of lymphatic system. Performing both procedure at a milder stage of disease could avoid also losing time and determining disease progression in patients at high risk. It has also to be stated that, in order to avoid overtreatment, we excluded from this approach patients affected by lymphedema stage I, in which the only LVAs can be performed with successful results (Yamamoto, Yamamoto, et al., 2016). Furthermore, performing the LVA at the same stage of VLNT, followed by SL after 2 weeks, offers patients an immediate improvement and also saves the patients an additional operation with the associated time off from work and recovery time rather than perform another surgery secondarily, as suggested by a recent studies (Chang et al., 2020; Cheng et al., 2014; de Sire et al., 2020).

Nevertheless, from the results of our study, we cannot state which procedure was more responsible for the results in patients treated with the combined approach. A patient in the VLNT and LVA group could have improved either from performing LVAs itself either from the combined synergy between LVA and VLNT. However, patients in the group I scored lower levels of reduction of the outcomes, proving that, whatever the responsible mechanism, LVA add a benefit for the patients. We acknowledge that studies with larger population and longer follow-up are needed to confirm our findings. Our study is ongoing and plans to enlarge our population are currently made. 


\section{5 | CONCLUSIONS}

In this pilot study, we evaluated for the first time the association of VLNT and LVAs in the same surgical procedure, followed by SL. LVA and VLNT can be integrated together in a combined approach to work in synergy, while enhancing the outcomes. SL following physiological procedures could help reducing the lymphatic load on the limb.

\section{ORCID}

\section{Giuseppe Di Taranto (D) https://orcid.org/0000-0002-3014-2419}

Alberto Bolletta (D) https://orcid.org/0000-0002-6418-2873

Luigi Losco (iD https://orcid.org/0000-0001-9105-1839

Rossella Elia (iD https://orcid.org/0000-0002-6094-5159

\section{REFERENCES}

Agko, M., Ciudad, P., \& Chen, H.-C. (2018). Staged surgical treatment of extremity lymphedema with dual gastroepiploic vascularized lymph node transfers followed by suction-assisted lipectomy-A prospective study. Journal of Surgical Oncology, 117(6), 1148-1156. https://doi. org/10.1002/jso.24969

Allen, R. J., \& Cheng, M.-H. (2016). Lymphedema surgery: Patient selection and an overview of surgical techniques. Journal of Surgical Oncology, 113(8), 923-931. https://doi.org/10.1002/jso.24170

Avraham, T., Yan, A., Zampell, J. C., Daluvoy, S. V., Haimovitz-Friedman, A., Cordeiro, A. P., \& Mehrara, B. J. (2010). Radiation therapy causes loss of dermal lymphatic vessels and interferes with lymphatic function by TGF-beta1-mediated tissue fibrosis. American Journal of Physiology. Cell Physiology, 299(3), C589-C605. https://doi.org/10.1152/ajpcell. 00535.2009

Ayestaray, B., Bekara, F., \& Andreoletti, J.-B. (2013). Patent blue-enhanced lymphaticovenular anastomosis. Journal of Plastic, Reconstructive \& Aesthetic Surgery, 66(3), 382-389. https://doi.org/10.1016/j.bjps. 2012.10.019

Becker, C., Assouad, J., Riquet, M., \& Hidden, G. (2006). Postmastectomy lymphedema: Long-term results following microsurgical lymph node transplantation. Annals of Surgery, 243(3), 313-315. https://doi.org/ 10.1097/01.sla.0000201258.10304.16

Bolletta, A., Di Taranto, G., Chen, S. H., Elia, R., Amorosi, V., Chan, J. C., \& Chen, H. C. (2020). Surgical treatment of Milroy disease. Journal of Surgical Oncology, 121(1), 175-181. https://doi.org/10.1002/jso. 25583

Brorson, H. (2004). Adipose tissue in lymphedema: The ignorance of adipose tissue in lymphedema. Lymphology, 37(4), 175-177.

Brorson, H. (2016). Liposuction in lymphedema treatment. Journal of Reconstructive Microsurgery, 32(1), 56-65. https://doi.org/10.1055/s0035-1549158

Casella, D., Di Taranto, G., Marcasciano, M., Sordi, S., Kothari, A., Kovacs, T., ... Ribuffo, D. (2019). Evaluation of Prepectoral implant placement and complete coverage with TiLoop bra mesh for breast reconstruction: A prospective study on long-term and patientreported BREAST-Q outcomes. Plastic and Reconstructive Surgery, 143 (1), 1e-9e. https://doi.org/10.1097/PRS.0000000000005078

Chang, D. W., Masia, J., Garza, R., Skoracki, R., \& Neligan, P. C. (2016). Lymphedema: Surgical and medical therapy. Plastic and Reconstructive Surgery, 138(3 Suppl), 209S-218S. https://doi.org/10.1097/PRS. 0000000000002683

Chang, E. I., Ibrahim, A., Liu, J., Robe, C., Suami, H., Hanasono, M. M., \& Nguyen, A. T. (2020). Optimizing quality of life for patients with breast cancer related lymphedema: A prospective study combining DIEP flap breast reconstruction and lymphedema surgery. Plastic and Reconstructive Surgery, 145, 676e-685e. https://doi.org/10.1097/PRS. 0000000000006634
Chen, H. C., O'Brien, B. M., Pribaz, J. J., \& Roberts, A. H. (1988). The use of tonometry in the assessment of upper extremity lymphoedema. British Journal of Plastic Surgery, 41(4), 399-402. https://doi.org/10.1016/ 0007-1226(88)90081-1

Cheng, M.-H., Chang, D. W., Masia, J., \& Koshima, I. (2020). Introduction of the 8th world symposium for lymphedema surgery. Journal of Surgical Oncology, 121(1), 7. https://doi.org/10.1002/jso.25620

Cheng, M.-H., Huang, J.-J., Nguyen, D. H., Saint-Cyr, M., Zenn, M. R., Tan, B. K., \& Lee, C. L. (2012). A novel approach to the treatment of lower extremity lymphedema by transferring a vascularized submental lymph node flap to the ankle. Gynecologic Oncology, 126(1), 93-98. https://doi.org/10.1016/j.ygyno.2012.04.017

Cheng, M.-H., Huang, J.-J., Wu, C.-W., Yang, C. Y., Lin, C. Y., Henry, S. L., \& Kolios, L. (2014). The mechanism of vascularized lymph node transfer for lymphedema: Natural lymphaticovenous drainage. Plastic and Reconstructive Surgery, 133(2), 192e-198e. https://doi.org/10.1097/ 01.prs.0000437257.78327.5b

Cheng, M.-H., Loh, C. Y. Y., \& Lin, C.-Y. (2018). Outcomes of vascularized lymph node transfer and Lymphovenous anastomosis for treatment of primary lymphedema. Plastic and Reconstructive Surgery. Global Open, 6 (12), e2056. https://doi.org/10.1097/GOX.0000000000002056

Ciudad, P., Agko, M., Huang, T. C. T., Manrique, O. J., Chang, W. L., Nicoli, F., ... Chen, H. C. (2019). Comprehensive multimodal surgical treatment of end-stage lower extremity lymphedema with toe management: The combined Charles', Homan's, and vascularized lymph node transfer (CHAHOVA) procedures. Journal of Surgical Oncology, 119(4), 430-438. https://doi.org/10.1002/jso.25356

Ciudad, P., Agko, M., Perez Coca, J. J., Manrique, O. J., Chang, W. L., Nicoli, F., ... Chen, H. C. (2017). Comparison of long-term clinical outcomes among different vascularized lymph node transfers: 6-year experience of a single center's approach to the treatment of lymphedema. Journal of Surgical Oncology, 116(6), 671-682. https://doi.org/ 10.1002/jso.24730

Ciudad, P., Manrique, O. J., Bustos, S. S., Coca, J. J. P., Chang, C. C., Shih, P. K., ... Chen, H. C. (2020). Comparisons in long-term clinical outcomes among patients with upper or lower extremity lymphedema treated with diverse vascularized lymph node transfer. Microsurgery, 40(2), 130-136. https://doi.org/10.1002/micr.30508

Ciudad, P., Manrique, O. J., Date, S., Agko, M., Perez Coca, J. J., Chang, W. L., ... Chen, H. C. (2017). Double gastroepiploic vascularized lymph node tranfers to middle and distal limb for the treatment of lymphedema. Microsurgery, 37(7), 771-779. https://doi.org/10.1002/ micr.30168

Cuzzone, D. A., Weitman, E. S., Albano, N. J., Ghanta, S., Savetsky, I. L., Gardenier, J. C., ... Mehrara, B. J. (2014). IL-6 regulates adipose deposition and homeostasis in lymphedema. American Journal of Physiology. Heart and Circulatory Physiology, 306(10), H1426-H1434. https://doi. org/10.1152/ajpheart.01019.2013

de Sire, A., Losco, L., Cigna, E., Lippi, L., Gimigliano, F., Gennari, A., ... Invernizzi, M. (2020). Three-dimensional laser scanning as a reliable and reproducible diagnostic tool in breast cancer related lymphedema rehabilitation: A proof-of-principle study. European Review for Medical and Pharmacological Sciences, 24(8), 4476-4485. https://doi.org/10. 26355/eurrev_202004_21030

Di Taranto, G., Chen, S.-H., Elia, R., Bolletta, A., Amorosi, V., Sitpahul, N., ... Chen, H. C. (2020). Free gastroepiploic lymph nodes and omentum flap for treatment of lower limb ulcers in severe lymphedema: Killing two birds with one stone. Journal of Surgical Oncology, 121(1), 168-174. https://doi.org/10.1002/jso.25581

Di Taranto, G., Chen, S.-H., Elia, R., Sitpahul, N., Chan, J. C. Y., Losco, L., ... Chen, H. C. (2019). Outcomes following head neck free flap reconstruction requiring interposition vein graft or vascular bridge flap. Head \& Neck, 41(9), 2914-2920. https://doi.org/10.1002/hed.25767

Di Taranto, G., Elia, R., Amorosi, V., Alamouti, R., Sitpahul, N., Chan, J., ... Chen, H. C. (2018). The difference in the caliber of efferent lymphatic 
vessels among various lymph node flaps. Journal of Surgical Oncology, 118(7), 1212-1213. https://doi.org/10.1002/jso.25257

Fernández Peñuela, R., Casaní Arazo, L., \& Masiá Ayala, J. (2019). Outcomes in vascularized lymph node transplantation in rabbits: A reliable model for improving the surgical approach to lymphedema. Lymphatic Research and Biology, 17, 413-417. https://doi.org/10.1089/Irb.2018. 0038

Fino, P., Di Taranto, G., Toscani, M., \& Scuderi, N. (2016). Surgical therapy of breast hypertrophy: A comparison of complications and satisfaction rate in large and small superior pedicle custom-made reduction mammaplasty. European Review for Medical and Pharmacological Sciences, 20(21), 4411-4415.

Hayashi, A., Hayashi, N., Yoshimatsu, H., \& Yamamoto, T. (2018). Effective and efficient lymphaticovenular anastomosis using preoperative ultrasound detection technique of lymphatic vessels in lower extremity lymphedema. Journal of Surgical Oncology, 117(2), 290-298. https:// doi.org/10.1002/jso.24812

Inserra, I., Martelli, C., Cipollina, M., Cicione, C., lavarone, F., di Taranto, G., ... Lattanzi, W. (2016). Lipoaspirate fluid proteome: A preliminary investigation by LC-MS top-down/bottom-up integrated platform of a high potential biofluid in regenerative medicine. Electrophoresis, 37 (7-8), 1015-1026. https://doi.org/10.1002/elps.201500504

International Society of Lymphology. (2013). The diagnosis and treatment of peripheral lymphedema: 2013 consensus document of the International Society of Lymphology. Lymphology, 46(1), 1-11.

Ito, R., Zelken, J., Yang, C.-Y., Lin, C.-Y., \& Cheng, M.-H. (2016). Proposed pathway and mechanism of vascularized lymph node flaps. Gynecologic Oncology, 141(1), 182-188. https://doi.org/10.1016/j.ygyno.2016. 01.007

Kaciulyte, J., Losco, L., Maruccia, M., Delia, G., Lo Torto, F., di Taranto, G., ... Cigna, E. (2019). Postsurgical antithrombotic therapy in microsurgery: Our protocol and literature review. European Review for Medical and Pharmacological Sciences, 23(10), 4448-4457. https://doi.org/10. 26355/eurrev_201905_17955

Lin, C.-H., Ali, R., Chen, S.-C., Wallace, C., Chang, Y. C., Chen, H. C., \& Cheng, M. H. (2009). Vascularized groin lymph node transfer using the wrist as a recipient site for management of postmastectomy upper extremity lymphedema. Plastic and Reconstructive Surgery, 123(4), 1265-1275. https://doi.org/10.1097/PRS.0b013e31819e6529

Lo Torto, F., Parisi, P., Casella, D., Di Taranto, G., Cigna, E., \& Ribuffo, D. (2018). Impact of evolving radiation therapy techniques on implantbased breast reconstruction. Plastic and Reconstructive Surgery, 141(1), 182e-183e. https://doi.org/10.1097/PRS.0000000000003972

Loh, C. Y. Y., Wu, J. C.-W., Nguyen, A., Dayan, J., Smith, M., Masia, J., ... Cheng, M. H. (2017). The 5th world symposium for lymphedema surgery-recent updates in lymphedema surgery and setting up of a global knowledge exchange platform. Journal of Surgical Oncology, 115 (1), 6-12. https://doi.org/10.1002/jso.24341

Losco, L., Roxo, A. C., Roxo, C. W., Lo Torto, F., Bolletta, A., de Sire, A., ... Roxo, C. P. (2020). Lower body lift after bariatric surgery: 323 consecutive cases over 10-year experience. Aesthetic Plastic Surgery, 44(2), 421-432. https://doi.org/10.1007/s00266-019-01543-x

Maeda, T., Yamamoto, Y., Iwasaki, D., Hayashi, T., Funayama, E., Oyama, A., ... Furukawa, H. (2018). Lymphatic reconnection and restoration of lymphatic flow by nonvascularized lymph node transplantation: Real-time fluorescence imaging using Indocyanine green and fluorescein Isothiocyanate-dextran. Lymphatic Research and Biology, 16 (2), 165-173. https://doi.org/10.1089/Irb.2016.0070

Maruccia, M., Pezzolla, A., Nacchiero, E., Dicillo, P., Macchia, L., Fiore, P., .. Elia, R. (2019). Efficacy and early results after combining laparoscopic harvest of double gastroepiploic lymph node flap and active physiotherapy for lower extremity lymphedema. Microsurgery, 39(8), 679-687. https://doi.org/10.1002/micr.30511

Masià, J., Pons, G., \& Rodríguez-Bauzà, E. (2016). Barcelona lymphedema algorithm for surgical treatment in breast cancer-related lymphedema.
Journal of Reconstructive Microsurgery, 32(5), 329-335. https://doi. org/10.1055/s-0036-1578814

Mihara, M., Hara, H., Hayashi, Y., Narushima, M., Yamamoto, T., Todokoro, T., ... Koshima, I. (2012). Pathological steps of cancerrelated lymphedema: Histological changes in the collecting lymphatic vessels after lymphadenectomy. PLoS One, 7(7), e41126. https://doi. org/10.1371/journal.pone.0041126

Patel, K. M., Lin, C.-Y., \& Cheng, M.-H. (2015). A prospective evaluation of lymphedema-specific quality-of-life outcomes following vascularized lymph node transfer. Annals of Surgical Oncology, 22(7), 2424-2430. https://doi.org/10.1245/s10434-014-4276-3

Phillips, G. S. A., Gore, S., Ramsden, A., \& Furniss, D. (2019). Lymphaticovenular anastomosis improves quality of life and limb volume in patients with secondary lymphedema after breast cancer treatment. The Breast Journal, 25(5), 859-864. https://doi.org/10.1111/tbj. 13348

Pons, G., Clavero, J. A., Alomar, X., Rodríguez-Bauza, E., Tom, L. K., \& Masia, J. (2019). Preoperative planning of lymphaticovenous anastomosis: The use of magnetic resonance lymphangiography as a complement to indocyanine green lymphography. Journal of Plastic, Reconstructive \& Aesthetic Surgery, 72(6), 884-891. https://doi.org/10. 1016/j.bjps.2019.02.024

Qiu, S. S., Chen, H.-Y., \& Cheng, M.-H. (2014). Vascularized lymph node flap transfer and lymphovenous anastomosis for klippel-trenaunay syndrome with congenital lymphedema. Plastic and Reconstructive Surgery. Global Open, 2(6), e167. https://doi.org/10.1097/GOX. 0000000000000099

Rockson, S. G., \& Rivera, K. K. (2008). Estimating the population burden of lymphedema. Annals of the New York Academy of Sciences, 1131, 147-154. https://doi.org/10.1196/annals.1413.014

Saaristo, A. M., Niemi, T. S., Viitanen, T. P., Tervala, T. V., Hartiala, P., \& Suominen, E. A. (2012). Microvascular breast reconstruction and lymph node transfer for postmastectomy lymphedema patients. Annals of Surgery, 255(3), 468-473. https://doi.org/10.1097/SLA. Ob013e3182426757

Seki, Y., Kajikawa, A., Yamamoto, T., Takeuchi, T., Terashima, T., \& Kurogi, N. (2019). The dynamic-lymphaticovenular anastomosis method for breast cancer treatment-related lymphedema: Creation of functional lymphaticovenular anastomoses with use of preoperative dynamic ultrasonography. Journal of Plastic, Reconstructive \& Aesthetic Surgery, 72(1), 62-70. https://doi.org/10.1016/j.bjps.2018. 09.005

Tarallo, M., Fino, P., Ribuffo, D., Casella, D., Toscani, M., Spalvieri, C., ... di Taranto, G. (2018). Liposuction aspirate fluid adipose-derived stem cell injection and secondary healing in fingertip injury: A pilot study. Plastic and Reconstructive Surgery, 142(1), 136-147. https://doi.org/10.1097/ PRS.0000000000004506

Viitanen, T. P., Mäki, M. T., Seppänen, M. P., Suominen, E. A., \& Saaristo, A. M. (2012). Donor-site lymphatic function after microvascular lymph node transfer. Plastic and Reconstructive Surgery, 130(6), 1246-1253. https://doi.org/10.1097/PRS.0b013e31826d1682

Yamamoto, T., \& Koshima, I. (2015). Supermicrosurgical anastomosis of superficial lymphatic vessel to deep lymphatic vessel for a patient with cellulitis-induced chronic localized leg lymphedema. Microsurgery, 35 (1), 68-71. https://doi.org/10.1002/micr.22327

Yamamoto, T., Yamamoto, N., Fuse, Y., Narushima, M., \& Koshima, I. (2018). Optimal sites for Supermicrosurgical Lymphaticovenular anastomosis: An analysis of lymphatic vessel detection rates on 840 surgical fields in lower extremity lymphedema patients. Plastic and Reconstructive Surgery, 142(6), 924e-930e. https://doi.org/10.1097/PRS. 0000000000005042

Yamamoto, T., Yamamoto, N., Yamashita, M., Furuya, M., Hayashi, A., \& Koshima, I. (2016). Efferent lymphatic vessel anastomosis: Supermicrosurgical efferent lymphatic vessel-to-venous anastomosis for the prophylactic treatment of subclinical lymphedema. Annals 
of Plastic Surgery, 76(4), 424-427. https://doi.org/10.1097/SAP. 0000000000000381

Yamamoto, T., Yoshimatsu, H., \& Yamamoto, N. (2016). Complete lymph flow reconstruction: A free vascularized lymph node true perforator flap transfer with efferent lymphaticolymphatic anastomosis. Journal of Plastic, Reconstructive \& Aesthetic Surgery, 69(9), 1227-1233. https://doi.org/10.1016/j.bjps.2016.06.028

Yap, Y. L., Lim, J., Shim, T. W. H., Naidu, S., Ong, W. C., \& Lim, T. C. (2009). Patent blue dye in lymphaticovenular anastomosis. Annals of the Academy of Medicine, Singapore, 38(8), 704-706.

Zampell, J. C., Yan, A., Elhadad, S., Avraham, T., Weitman, E., \& Mehrara, B. J. (2012). CD4(+) cells regulate fibrosis and lymphangiogenesis in response to lymphatic fluid stasis. PLoS One, 7 (11), e49940. https://doi.org/10.1371/journal.pone.0049940

How to cite this article: $\mathrm{Di}$ Taranto G, Bolletta A, Chen S-H, et al. A prospective study on combined lymphedema surgery: Gastroepiploic vascularized lymph nodes transfer and lymphaticovenous anastomosis followed by suction lipectomy. Microsurgery. 2020;1-10. https://doi.org/10.1002/micr.30641 